\title{
Why Non-Specific Adaptation Mechanisms Inevitably Lead to the Emergence of Bacterial Strains Multiresistant to Antibiotics
}

\author{
Marcos López-Pérez* \\ Department of Environmental Sciences, Autonomous Metropolitan University, Mexico
}

Received: February 23, 2018; Published: March 08, 2018

*Corresponding author: Marcos López Pérez, Department of Environmental Sciencies, Autonomous Metropolitan University, Lerma de Villada, Mexico, Email: m.lopez@correo.ler.uam.mx

Keywords: Non-specific adaptation mechanisms; strains multiresistants; Antibiotics

\section{Introduction}

The resistance to antibiotics phenomenon has become a public health problem in the world [1]. In addition, it is interesting to highlight that bacterial infectious diseases are the cause of a high mortality rate in the world [2] which sets up a scenario of enormous gravity. A clear example is which already have been reported Klebsiella pneumonistrains, resistant to all available antibiotics [3]. This phenomenon has increased its severity by several factors, among which we can highlight.

a. The population increment, which leads to a greater number of patients under therapy with antibiotics.

b. The decrease of development of new antimicrobial drugs, as a result, the selection pressure that is exerted on the pathogenic microorganisms is maintained over time.

c. The inappropriate use of these drugs, that leads recurrently to these pathogenic bacteria come into contact with the antibiotics under sub lethal concentrations and finally.

d. The mobilityrate increase of resistance mechanisms between environmental bacteria carriers of resistance and susceptible bacteria with pathogenic capacity.

The unitary or synergistic effect of these factors has as unfailing consequence, the emergence of resistant bacterial strains. For the development of new strategies and a comprehensive understanding of this problem, it is necessary to consider the evolutionary adaptive dynamics perspective and consequently leading to this scenario should be the understanding not only from a clinical perspective, but should also involve a broader theoretical framework, the Microbial Ecology. In this sense, there are already several published papers that are highly relevant, that focus on the analysis of the resistance mechanisms considering mainly the ecology of bacteria, that is highlight the role of antibiotics not only as a causative of bacterial lysis or bacteriostaticagent, but also it considers elements involved in cellular communication, stress, virulence, aggregation for biofilmsformation [4-5].

This approach facilitates the understanding of the mechanisms that confer resistance, as shown in (Figure 1). These mechanisms may result in a degree of total or partial resistance, which is related to the specific nature of the resistance mechanism, for example the resistance that is developed as a result of the chemical modification of an antibiotic is total, since it inhibits the potential interactions with the target in the bacteria physiology, However, other resistance mechanisms confers partial resistance, but for this, the process involves decrease by different procedures the interactions between target and the antibiotic. One of these mechanisms has already been reported as one of particular importance to explain the emergence of superbugs' strains is the cellular efflux [6]. The main feature to adaptive purposes is that they are inherently nonspecific and therefore are promiscuous, understanding that they are able to interact with very different nature elements [7]. On 
the other hand, it has been determined that are expressed in the bacterial physiology as primary response to any kind of stress. In this sense, it has also been reported that some of the interesting features in multiresistant strains is the overexpression of these mechanisms [8]. It can be understood as the result of selection pressure intensification due to of the increase in two parameters

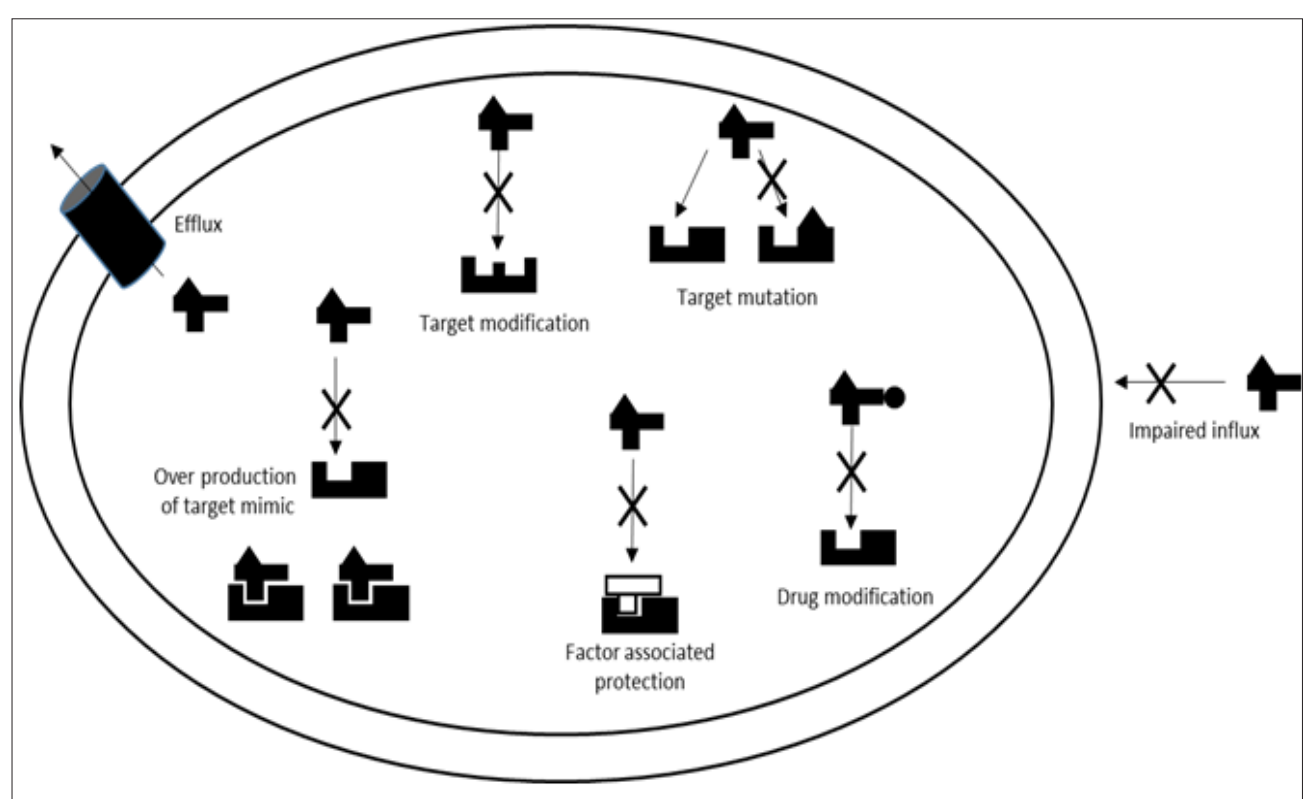

Figure 1: Antibacterial resistance mechanisms. Image modified from Ribosome-targeting antibiotics and bacterial resistance mechanisms Nature Reviews Microbiology Daniel N. Wilson [11].

a) The number

b) Diversity of drugs with antimicrobial activity in microbial ecosystems related to human activity.

c) Even in the ecosystem where the selection pressure is more intense, the intestinal tract of patients under therapy with antibiotics. In addition to cellular efflux, bacteria developed other mechanisms of unspecified adaptation:

d) Maximize the rate of sequences exchange through horizontal gene transfer; in this sense it has already been demonstrated that the presence of antibiotics promotes the exchange [9]. It would be interesting to assess if the concentration of an antibiotic depends on the rate of exchange.

e) Avoid plank tonic cell shape, more isolated and therefore more exposed in these environments.

f) Finally a nonspecific mechanism would focus on increasing the rate of mutations, as suggested by the work of Martinez and Baquero [10-11].

\section{Conclusion}

A clear conclusion that it is possible to infer is that the bacteria have shown an enormous adaptive versatility to all kinds of factors and circumstances in the evolutionary history. On the other hand, antibiotics have proven to be a good tool for the control of bacterial sepsis, however, this clear trend toward the pathogenic strains multiresistants emergence should lead to rethink this strategy, and consequently new therapies must consider other organisms that have co-evolved with the bacteria and that also demonstrated to have an enormous adaptive adaptation, since after millions of years sharing the same ecological niche are capable to kill bacteria, the bacteriophages.

\section{References}

1. Prestinaci F, Pezzotti P, Pantosti A (2015) Antimicrobial resistance: a global multifaceted phenomenon. Pathogens and Global Health 109(7): 309-318.

2. Dye C (2014) After 2015: infectious diseases in a new era of health and development. Philosophical Transactions of the Royal Society B: Biological Sciences 369(1645): 20130426.

3. Paczosa MK, Mecsas J (2016) Klebsiella pneumoniae: Going on the Offense with a Strong Defense. Microbiology and Molecular Biology Reviews 80(3): 629-661.

4. Sengupta S, Chattopadhyay MK, Grossart HP (2013) The multifaceted roles of antibiotics and antibiotic resistance in nature. Frontiers in Microbiology 12(4): 47.

5. Jose L Martinez (2009) The role of natural environments in the evolution of resistance traits in pathogenic bacteria. Proc R Soc B 276: 2521-2530.

6. Blanco P, Hernando-Amado S, Reales-Calderon JA (2016) Bacterial Multidrug Efflux Pumps: Much More Than Antibiotic Resistance Determinants. Poirel L ed Microorganisms 4(1): 14.

7. Jingjing Sun, Ziqing Deng, Aixin Yan (2014) Bacterial multidrug efflux pumps: Mechanisms, physiology and pharmacological exploitations. Biochemical and Biophysical Research Communications 453: 254-267.

8. Koo SH (2015) Overexpression of Efflux Pump in Multiresistant Pseudomonas aeruginosa: How You Will Discover and Treat It? Infection \& Chemotherapy 47(2): 142-144.

9. Charpentier X, Polard P, Claverys JP (2012) Induction of competence for genetic transformation by antibiotics: convergent evolution of stress responses in distant bacterial species lacking SOS? Curr Opin Microbiol 15: $570-576$. 
10. Martinez JL, Baquero F (2000) Mutation frequencies and antibiotic resistance. Antimicrob Agents Chemother 44(7): 1771-1777.

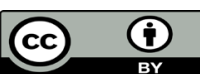

This work is licensed under Creative Commons Attribution 4.0 License

Submission Link: https://biomedres.us/submit-manuscript.php
11. Daniel N Wilson (2014) Ribosome-targeting antibiotics and mechanisms of bacterial resistance. Nature Reviews Microbiology 12: 35-48.

$\begin{array}{ll}\text { BIOMEDICAL } & \text { Assets of Publishing with us } \\ \text { RESEARCHES } & \text { - Global archiving of articles } \\ \text { - Immediate, unrestricted online access }\end{array}$

